\title{
Volatility Estimation via Jump Indicator
}

\author{
R. Aboulaich ${ }^{1,2}$, H. Ben Ameur ${ }^{3,4}$ \& M. Lamarti Sefian ${ }^{1,2}$ \\ ${ }^{1}$ LERMA, Mohammadia School of Engineers, Avenue Ibn Sina B.P 765, Agdal, Rabat, Maroc \\ 2 LIRIMA http://www.lirima.uninet.cm \\ ${ }^{3}$ LAMSIN, National School of Engineers of Tunis, Campus universitaire 2092 Manar II, Tunis \\ ${ }^{4}$ IPEST, Institut Préparatoire aux études scientifiques et technique, route Sidi Bou Said, B.P 51, 2075 LaMarsa, \\ Tunis \\ Correspondence: R. Aboulaich, LERMA, Mohammadia School of Engineers, Avenue Ibn Sina B.P 765, Agdal, \\ Rabat, Maroc. E-mail: aboulaich@emi.ac.ma
}

Received: September 25, 2013 Accepted: February 10, 2014 Online Published: February 17, 2014

doi:10.5539/mas.v8n2p12 URL: http://dx.doi.org/10.5539/mas.v8n2p12

\begin{abstract}
The volatility is considered constant in Black and Scholes model. However, this implausible assumption leads to an undervaluation of options. We try to remediate to this drawback considering a more realistic model where the volatility is a piecewise constant function of time. We introduce a jump indicator to locate iteratively discontinuities of volatility and use an optimization process to estimate volatility values. We compare our results with regularization method (Aboulaich \& Medarhri, 2013) and "AutoRegressive Conditional Heteroskedasticity" ARCH method (Engle, 1982).
\end{abstract}

Keywords: inverse problem, volatily estimation, jump indicator, lookback option

\section{Introduction}

An inverse problem of parameter estimation consists in identifying unknown parameters which are space dependent coefficients in a system of partial differential equations modeling a physical problem. We are interested in volatility estimation in a finance model.

The price of an option (prime) depends on the volatility $\sigma$. We consider the case of lookback option using a Black and Scholes PDE studied in (Aboulaich, Alami Idrissi, \& Lamarti, 2012).

The Black and Scholes (B\&S) model for the evaluation of an option price, is given by the following stochastic equation (Aboulaich et al., 2012):

$$
d S_{t}=\mu d t S_{t}+\sigma S_{t} d B_{t}
$$

where $\mu$ is the drift rate, $\sigma \in \mathbb{R}^{+}$is the volatility which is considered constant and $B_{t}$ follows a Brownian motion. Stochastic integration of this equation gives the analytical solution of the underlying asset which is given by:

$$
S_{t}=S_{0} \exp \left(\left[r-\frac{\sigma^{2}}{2}\right] t+\sigma \varepsilon \sqrt{d t}\right)
$$

where $r$ is the short rate of interest, $S_{0}$ is the underlying price as $t=0$.

The price of the call option $P$ (Prime) is obtained using the following equation:

$$
P(t)=e^{-r(T-t)} E\left(S_{t}\right)
$$

Where $T$ is the expiration date of the option (Maturity) and $E\left(S_{t}\right)$ is the expectation of the underlying price. 
Using Itô's Lemma (Itô, 1942), the prime $P$ for an option satisfies Black and Scholes model given by the Partial Differential Equation:

$$
\frac{\partial P}{\partial t}(S, M, t)+\frac{1}{2} \sigma(S, t)^{2} S^{2} \frac{\partial^{2} P}{\partial S^{2}}(S, M, t)+r S \frac{\partial P}{\partial S}(S, M, t)-r P(S, M, t)=0
$$

where $P$ is the prime, $S$ is the price of the underlying asset, $r$ is the rate that is considered constant and $M$ is the maximum price of $S$ obtained before the maturity.

A crucial assumption in the B\&S model is that the volatility is constant. This non realistic assumption lead, in particular application, to undervaluation of European option prices (Deberse, 2006).

The volatility jump is a normal phenomena which can be a consequence of an intrinsic new information that disrupt the market, or a consequence of the behavior of the volatility itself (increasing or decreasing).

Some models take in account jumps of volatility values (Aboulaich et al., 2012; Cox, Ingersoll, \& Ross, 1985; Dupire, 1994; Engle, 1982; Merton, 1976); we cite as example the Merton model (Merton, 1976) where the prime $P$ is computed via Poisson distribution. Using a finite element method, $P$ is computed considering discrete discontinuities (Aboulaich et al., 2012). Other studies consider the volatility as a continuous function of the time; we cite as example the ARCH method (Engle, 1982); we can find also models that volatility depends of asset prices; Cox and Ross (1985) or as a function of asset price and time (Dupire, 1994).

We consider a deterministic model, simple to implement, and we develop an algorithm allowing to locate the volatility discontinuities and to estimate different values of the volatility that we suppose a piecewise constant function of time. The idea is to proceed in an iterative way to identify jointly the positions of discontinuities in the time interval where the model is defined and volatility values. This idea was initially developed for the estimation of hydraulic transmissivity in porous media (Benameur, Chavent, \& Jaffré, 2002; Benameur, Chavent, Clément, \& Weis, 2008) An adaptive parameterization algorithm guided by refinement indicators is developed and tested on several parameterizations. We develop an algorithm for volatility estimation using jump indicators in a similar way as refinement indicators and an optimization process to compute volatility values. We introduce the jump indicator in section 3. We compare the results of our algorithm with those obtained using Tikhonov regularization method developed for the Dupire model in (Aboulaich \& Medarhri, 2013) and introduced by (Chiarella, Craddock, \& el Hassan, 2007) for the Black and Scholes model, and also with results obtained with ARCH method for the stock market index Standard \& Poor's 500 (S\& P500).

\section{Inverse Problem}

The volatility estimation is an inverse problem of parameter estimation. The parameter estimation problem can be set as a minimization problem of an objective function defined as a misfit between measurements and the corresponding quantities computed with a considered parameter (solution of the partial differential equations system under consideration).

We assume that the volatility is a piecewise constant function of time and we formulate the inverse problem of identifying the parameter volatility as an optimization problem. We consider an objective function $J$ defined as a misfit between observed values of prime and the computed prime values, via Equation 4, considering a current volatility $\sigma$.

$$
\min _{\sigma} J(\sigma, P)=\frac{1}{2}\left\|P(\sigma)-P_{o b s}\right\|^{2}
$$

Where $P_{o b s}$ is the market data (prime observations) and $P(\sigma)$ is the solution of 4 considering a volatility $\sigma$.

For the numerical resolution of this problem, we introduce the Lagrangian associated with the minimization problem 5 with the constraint 4

$$
L(\sigma, P, q)=J(\sigma, P)-\int_{\Omega}\left(\frac{\partial P}{\partial t}+\frac{1}{2} \sigma^{2} S^{2} \frac{\partial^{2} P}{\partial S^{2}}+r \frac{\partial P}{\partial S}-r P\right) q d \Omega
$$

$\Omega=\{(S, M, t) \in[0,1] \times[0,1] \times[0, T] / S \leq M\}$ and $q \in H_{0}^{1}(\Omega)$ is the Lagrange multiplier. 
Let be $w \in H_{0}^{1}(\Omega)$, the optimality condition is :

$$
\frac{\partial L}{\partial P}(\sigma, P, q) . w=0, \quad \forall w \in H_{0}^{1}(\Omega)
$$

Using the derivative definition:

$$
\frac{\partial L}{\partial P} \cdot w=\lim _{\varepsilon \rightarrow 0} \frac{L(\sigma, P+\varepsilon w, q)-L(\sigma, P, q)}{\partial \varepsilon}
$$

we obtain

$$
\frac{\partial L}{\partial P} \cdot w=\frac{\partial J}{\partial P} \cdot w-\int_{\Omega}\left(\frac{\partial w}{\partial t}+\frac{1}{2} \sigma^{2} S^{2} \frac{\partial^{2} w}{\partial S^{2}}+r S \frac{\partial w}{\partial S}-r w\right) \cdot q, \forall w \in H_{0}^{1}(\Omega)
$$

Applying the Green formula twice and using the condition that the prime holds 0 at the maturity $T ; P(T)=0$, for all $w \in H_{0}^{1}(\Omega)$ and $q \in H_{0}^{1}(\Omega)$, we obtain:

$$
\frac{\partial L}{\partial P} \cdot w=\frac{\partial J}{\partial P} \cdot w+\int_{\Omega}\left(\frac{\partial q}{\partial t}-\frac{1}{2} \sigma^{2} S^{2} \frac{\partial^{2} q}{\partial S^{2}}+r S \frac{\partial q}{\partial S}+r q\right) w, \forall w \in H_{0}^{1}(\Omega)
$$

on the other hand, we have:

$$
\frac{\partial J}{\partial P} \cdot w=\int_{\Omega}\left|P-P_{o b s}\right| . w \forall w \in H_{0}^{1}(\Omega)
$$

Finally, we get the following adjoint equation:

$$
\int_{\Omega}\left(\left|P-P_{o b s}\right|+\frac{\partial q}{\partial t}-\frac{1}{2} \sigma^{2} S^{2} \frac{\partial^{2} q}{\partial S^{2}}+r S \frac{\partial q}{\partial S}+r q\right) w=0, \forall w \in H_{0}^{1}(\Omega)
$$

The PDE satisfied by the adjoint state is:

$$
\frac{\partial q}{\partial t}-\frac{1}{2} \sigma^{2} S^{2} \frac{\partial^{2} q}{\partial S^{2}}+r S \frac{\partial q}{\partial S}+r q=-\left|P-P_{o b s}\right|
$$

The second optimality condition is:

$$
\frac{\partial L}{\partial \sigma} \cdot \tau=\lim _{\varepsilon \rightarrow 0} \frac{L(\sigma+\varepsilon \tau, P, q)-L(\sigma, P, q)}{\partial \varepsilon}
$$

Denoting $\psi(\tau)=\frac{\partial P}{\partial \sigma}(S, M, \tau)$, and considering the state equation 4 we have:

$$
\frac{\partial L}{\partial \sigma} \cdot \tau=\frac{\partial J}{\partial \sigma} \cdot \tau-\int_{\Omega}\left(\frac{\partial \psi(\tau)}{\partial t}+\sigma S^{2} \frac{\partial^{2} P(S, M, \sigma)}{\partial S^{2}}+\frac{1}{2} \sigma^{2} S^{2} \frac{\partial^{2} \psi(\tau)}{\partial S^{2}}+r S \frac{\partial \psi(\tau)}{\partial S}-r \psi(\tau)\right) \cdot q, \quad \forall \tau \in[0, T]
$$

taking $w=\psi(\tau)$ in 6 , we obtain:

$$
\frac{\partial J}{\partial P} \cdot \psi(\tau)=\int_{\Omega}\left(\frac{\partial \psi(\tau)}{\partial t}+\frac{1}{2} \sigma^{2} S^{2} \frac{\partial^{2} \psi(\tau)}{\partial S^{2}}+r S \frac{\partial \psi(\tau)}{\partial S}-r \psi(\tau)\right) . q \forall \tau \in[0, T] ; \forall q \in H_{0}^{1}(\Omega)
$$

By identifying the Equations 11 and 12, we obtain:

$$
\frac{\partial L}{\partial P} \cdot \tau=\frac{\partial J}{\partial \sigma} \cdot \tau-\frac{\partial J}{\partial P} \cdot \psi(\tau)-\int_{\Omega} \sigma S^{2} \frac{\partial^{2} P(\sigma, S, M)}{\partial S^{2}} q
$$


and finally the optimality condition is:

$$
\frac{\partial J}{\partial \sigma} \cdot \tau=-\int_{\Omega} \sigma S^{2} \frac{\partial^{2} P(\sigma, S, M)}{\partial S^{2}} q
$$

In the following section we develop the idea of using this gradient as an indicator to detect the location of "relatively" important discontinuity of the volatility. Considering that the volatility is piecewise constant, this location is a location of volatility jump.

\section{Jump Indicator}

The inverse problem of parameter estimation is first set as the minimization of an objective function defined as a misfit between data and computed values considering a current parameter $\mathrm{p}$.

If $p_{\text {true }} \in P$ denotes the unknown parameter that we are looking for in a set of admissible parameters $P^{a d}$, and if $d \simeq \mathcal{F}\left(p_{\text {true }}\right) \in \mathbb{R}^{n}$ (the data) denotes the corresponding available measurements of the solution of the PDE, one can attempt an indirect determination of $p_{\text {true }}$ from $d$ by solving the

$$
\text { minimize } \mathcal{J}(p) \text { for } p \in P^{a d}
$$

where $\mathcal{J}(p)$ is the least-squares misfit between data $d$ and the corresponding quantities $\mathcal{F}(p)$ computed from the current parameter $p$,

$$
\mathcal{J}(p)=\frac{1}{2}\|d-\mathcal{F}(p)\|^{2} .
$$

$\mathcal{F}$ is an operator defined by the PDE and the unknown parameter is considered piecewise constant.

The notion of adaptive parameterization introduced by Benameur Chavent et Jaffré (BenAmeur et al., 2002) is induced by zonations of the domain in zones such that the parameter is constant in each zone. The decrease of the optimal value of the objective function resulting from the splitting of one zone into two subzones is then quantified for the linearized problem in the continuous case, and refinement indicators are defined, in the general case, from a first order approximation of the objective function variation.

Minimizing such an objective function avoids the difficulty of inverting $\mathcal{F}$, which is impossible in most cases.

In our case the unknown parameter is the volatility $\sigma$ that we suppose piecewise constant function of time and $\mathcal{F}$ is defined by the Black and Scholes model.

A similar idea is to define a Jump Indicator in order to define a partition of the domain $\Omega=\{(S, M, t) \in[0,1] \times$ $[0,1] \times[0, T] / S \leq M\}$ given by $\Omega_{1}=\left\{(S, M, t) \in[0,1] \times[0,1] \times\left[0, t_{1}\right] / S \leq M\right\}$ and $\Omega_{2}=\{(S, M, t) \in[0,1] \times[0,1] \times$ $\left.\left[t_{1}, T\right] / S \leq M\right\}$ such that the volatility is constant in each part $\Omega_{k}, k=1,2$. The method is based on a computation of an indicator that permits to locate iteratively the jumps. This indicator locates the volatility discontinuities which has the greater amplitude and more effect to the underlying and step by step we will try to locate the smaller amplitude jumps. To simplify the presentation, we explain the approach for one jump, the numerical tests are made for the case of two jumps in a synthetic example and three jumps for market data, the method can be generalized to multiple ones.

Let $\left(P_{1}\right)$ be the minimization problem considering the volatility is constant during the maturity. Therefore, a single parameter $\sigma_{0}^{*}$ will be estimated and that corresponds to the optimal solution of the minimization problem $J(\sigma)$. Let $\left(P_{2}\right)$ be the minimization problem of $J(\sigma)$ associated with a new partition $z_{1}$ and $z_{2}$ supposing that $\sigma$ has one discontinuity. The solution of the problem $\left(P_{2}\right)$ is presented as $J_{P_{2}}\left(\sigma_{1}^{*}, \sigma_{2}^{*}\right)$ with $\sigma_{1}^{*}$ corresponds to the area $z_{1}$ and $\sigma_{2}^{*}$ corresponds to the area $z_{2}$, we note $\sigma^{*}=\left(\sigma_{1}^{*}, \sigma_{2}^{*}\right)$.

To determine the discontinuity, we will consider the difference $c$ between the two parameters $\sigma_{1}$ and $\sigma_{2}$ and we consider the problem of minimization $J_{P_{2}}$ under constraint $c=\sigma_{2}^{*}-\sigma_{1}^{*}$.

$$
J_{P_{2}}\left(\sigma^{*}\right)=\min _{c} J_{P_{2}}(\sigma)
$$

If $c=0$, then the solution $J_{P_{2}}\left(\sigma^{*}\right)$ (with $\sigma^{*}=\left(\sigma_{0}^{*}, \sigma_{0}^{*}\right)$ ) corresponds to the first problem. In the other case, the optimal value of the problem under constraint $c$ corresponds to the optimum value of $J_{P_{2}}\left(\sigma_{1}^{*}, \sigma_{2}^{*}\right)$. 
If we note $J^{c}=J_{P_{2}}\left(\sigma_{1}^{*}, \sigma_{2}^{*}\right)$ and $J^{0}=J_{P_{2}}\left(\sigma_{0}^{*}, \sigma_{0}^{*}\right)$, using:

$$
J^{c}=J^{0}+\left.\frac{\partial J^{c}}{\partial c}\right|_{c=0} . c+O(c)
$$

The norm $\left\|\left.\frac{\partial J^{c}}{\partial c}\right|_{c=0}\right\|$ measures the variation in $J^{c}$ in relation to the difference between the two parameters $\sigma_{1}^{*}$ and $\sigma_{2}^{*}$ through $c$, this norm is the Jump Indicator.

On the other hand, we associate the minimization problem under constraint $c=\sigma_{2}^{*}-\sigma_{1}^{*}$ to the Lagrangian defined by:

$$
L_{c}(\sigma, \lambda)=J(\sigma)+\langle\lambda, A \sigma-c\rangle
$$

With $A=[-1.1]$ and $\lambda$ is the Lagrange multiplier associated with the constraint $c=\sigma_{2}^{*}-\sigma_{1}^{*}$.

The optimum value $\sigma^{*}=\left(\sigma_{1}^{*}, \sigma_{2}^{*}\right)$ verifies the following conditions.

At the optimum, we have:

$$
J_{c}^{*}=J\left(\sigma^{*}\right)=L_{c}\left(\sigma^{*}, \lambda^{*}\right)
$$

and

$$
\left.\frac{\partial L_{c}}{\partial c}\right|_{c=0}\left(\sigma^{*}, \lambda^{*}\right)=0
$$

Then:

$$
\left.\frac{\partial J_{c}^{*}}{\partial c}\right|_{c=0}-\lambda^{*}=0
$$

In our example, the Jump Indicator for the partition of $[0, T]$ is:

$$
I=\left|\lambda^{*}\right|=\left|\frac{\partial J}{\partial \sigma_{1}}\left(\sigma^{*}\right)\right|=\left|-\frac{\partial J}{\partial \sigma_{2}}\left(\sigma^{*}\right)\right|
$$

\section{Algorithm for the jump determination}

In order to simplify the presentation of the method, we will write the algorithm 1 in case of one jump. We generalize in the algorithm 2 for the case of multiple jumps. To determine the time of one jump, we will divide our domain $\Omega=\Omega_{1} \times[0, T]$ with $\Omega_{1}=\{(S, M) \in[0,1] \times[0,1] / S \leq M\}$ in two sub-domains $\Omega_{1} \times\left[0, t_{1}\right]$ and $\Omega_{1} \times\left[t_{1}, T\right]$, where $t_{1}$ is the moment and the norm $\left\|\frac{\partial J^{c}}{\partial c}\right\|$ is maximum.

We have for each time $t_{k}$, the Jump Indicator $I_{k}$ is given by:

$$
I_{k}=\left|\left(\sum_{t_{l}=0}^{k} \sum_{j=0}^{n}\left(\sum_{i=j}^{n}\left(\frac{\partial J}{\partial \sigma}\right)_{S_{i}, M_{j}, t_{l}}\right)\right)\right|
$$

where

- $S_{i}=i h$;

- $M_{j}=j h$;

- $h=\Delta S=\Delta M$;

- $t_{l}=l \Delta \mathrm{t}$.

In this case where we have only one jump, the algorithm is then given by:

\section{Algorithm: 1}

- For $k=1$ to a maximum time step.

- Resolution of the state equation to determine $P$;

- Calculation of $\nabla J^{*}$ from the condition of optimality.

- Computation of $I_{k}$.

- Return the Jump Indicator that corresponds to the max of $I_{k}$, and we calculate $\sigma_{1}$. 
In the following, we will present the algorithm in the case of multiple jumps. We will divide the domain $\Omega$ in a union of the domains $\Omega_{h}=\Omega_{1} \times\left[t_{h} ; t_{h+1}\right]$ :

$\Omega=\bigcup_{h=0}^{n} \Omega_{h}$ and $t_{0}=0$ et $t_{n}=T$, the algorithm will be:

\section{Algorithm: 2}

- repeat

- For $\mathrm{h}=1$ to $\mathrm{n}$;

- Call Alg 1 and we obtain $I_{k, h}$ and $\sigma_{k, h}$;

- Calculate $J_{h}\left(\sigma_{k, h}\right)$;

- Return $\sigma_{k, h *}$ and $I_{k, h *}$ who gives the minimum of $J_{h}$;

- $I_{k+1}=I_{k, h *}$ and $\sigma_{k+1}=\sigma_{k, h *}$;

- while $\left\|\sigma_{k+1}-\sigma_{k}\right\|<\epsilon$ or $\left\|I_{k}\right\| \mid<\epsilon$.

Before doing the numerical results and comparisons, we briefly recall the inverse method using a Tikhonov regularization (Chiarella et al., 2007) (section 4) and a statistical method using ARCH method (Engle, 1982) (section $5)$.

\section{Calibration of a Volatility by a Regularization Strategy}

Lagnado and Osher (Lagnado \& Osher, 1997) gave an estimate of volatility using the inverse problem associated with the Black-Scholes equation $\left(E_{1}\right)$. The problem is to minimize $G(\sigma)$ :

$$
G(\sigma)=\sum_{i=1}^{N} \sum_{j=1}^{M_{i}}\left[P\left(S_{0} ; 0 ; K_{i j} ; T_{i} ; \sigma\right)-P^{i j}\right]^{2}
$$

$M_{i}$ denotes the number of Strike, $T_{i}$ is the expiration date of an option and $K_{i j}$ is the Strike for the date $T_{i}$. This problem is ill-posed, regularization Tikhonov type (Tikhonov, 1963) was introduced as follows:

$$
F(\sigma)=\|\sigma\|_{2}^{2}+\lambda G(\sigma)
$$

However, the calibration strategy of Lagnado and Osher is made for one fixed value of $S_{0}$ at time $t=0$. Aboulaich et al proposed in (Aboulaich \& Medarhri, 2013) an algorithm to compute the volatility using the modified calibration strategy introduced by (Chiarella et al., 2007), actualizing the price of the option simultaneously with $S$ depending on the time $t$, the problem $G(\sigma)$ becomes:

$$
G(\sigma)=\sum_{i=1}^{N} \sum_{j=1}^{M_{i}}\left[P\left(S ; 0 ; K_{i j} ; T_{i} ; \sigma\right)-P^{i j}\right]^{2}
$$

In order to minimize this functional, the Euler-Lagrange equation is used. From the variation calculus, the volatility is obtained solving the following Poisson's Equation:

$$
\frac{\partial^{2} \sigma}{\partial S^{2}}+\frac{\partial^{2} \sigma}{\partial t^{2}}=\lambda w(\sigma)
$$

with

$$
w(\sigma)=\sum_{i=1}^{N} \sum_{j=1}^{M_{i}} \frac{\partial P}{\partial \sigma}\left(S ; 0 ; K_{i j} ; T_{i} ; \sigma\right)\left[P\left(S ; 0 ; K_{i j} ; T_{i} ; \sigma\right)-P^{i j}\right]
$$

For more details, see (Aboulaich \& Medarhri, 2013) and (Chiarella et al., 2007). 


\section{Algorithm presented in (Aboulaich \& Medarhri, 2013)}

To estimate the volatility, the following steps are used:

- Approach $\sigma_{0}$ by a function $\phi_{0}(S, t)$.

- For $k=0$ repeat

- Resolution of the Black and Scholes model to determine $P$ and compute $\frac{\partial P}{\partial \sigma}$;

- Computation of $w(\sigma(k))$ given by (22)

- Solve the Poisson's equation (21) to determinate $\sigma(k+1)$

$-k=k+1$

- Until $\|\sigma(k+1)-\sigma(k)\|<\epsilon$

The estimation of $\frac{\partial P}{\partial \sigma}\left(S, 0, K_{i j} T_{i} \sigma\right)$ is made by the finite differences method.

In order to compare our results with the statistical model, we use ARCH method presented in the next section.

\section{ARCH Models}

Auto Regressive Conditional Heteroscedasticity "ARCH" models are a stochastic processes which describe the variation of the second order moment. Introduced by Robert F. Engle (1982), it is based on the modeling of the conditional variance and identifies the presence of successive periods of volatility and relative stability. The $\operatorname{ARCH}(q)$ volatility model is described by:

$$
\left\{\begin{array}{l}
\epsilon_{i}=v_{i} \sqrt{\sigma_{i}} \\
\sigma_{i}^{2}=\alpha_{0}+\sum_{i=1}^{q} \alpha_{i} \epsilon_{t-i}
\end{array}\right.
$$

$v_{i}$ follows the normal distribution and $\left(\alpha_{i}\right)$ are the parameters model.

The estimation of these parameters can be made by the maximum likelihood method or the least Square method.

\section{Algorithm used for maximum likehood}

In order to use $\operatorname{ARCH}(q)$ model, we have to verify if the series have some properties; normality, linearity and heteroscedasticity (or not homoscedasticity):

- We use the portemanteau (Droesbeke, Fichet, \& Tassi,1989) test to verify linearity.

- The normality is given by the Jarque et Bera test (Bourbonnais, 2002).

- In order to verify the heteroscedasticity, we use the following test; which aims to determine if the variance of the chronological series changes over time:

- Null hypothesis $H_{0}$ (homoscedasticity) $\alpha_{1}=\alpha_{2}=\ldots=\alpha_{q}=0$;

- Alternative hypothesis: at least one non-zero value.

Based on the Lagrange Multiplier statistical $L M=\mathrm{NR}^{2}$ (Engle, 1982) which is distributed according to the law chi-square with degrees of freedom $p=2 q$.

$N$ is the size of the sample and $R^{2}$ is the coefficient of determination.

If LM is greater than the theoretical value of $\chi$, we reject the null hypothesis and then we can say that the model is heteroscedastic.

\section{Numerical Results}

We start by testing the algorithm 2 using synthetic data and determining the jumps corresponding to the discontinuities of the parameter. In a second test, we search for the jumps associated to the discontinuities considering the S\&P500 between 03/01/2011 and 25/05/2011. 


\subsection{Synthetic Example}

We generate prices from Black-Scholes model 2 using fictive data:

$$
S_{t}=S_{0} \exp \left(\left[r-\frac{\sigma^{2}}{2}\right] t+\sigma \varepsilon \sqrt{d t}\right)
$$

where $\varepsilon$ follows the normal process.

To locate the discontinuities, we consider a partition into zones $Z_{i}$ with $\sigma$ constant on each one. We apply the methods described in section 3 and 4 to find these discontinuities and calculate $\sigma$.
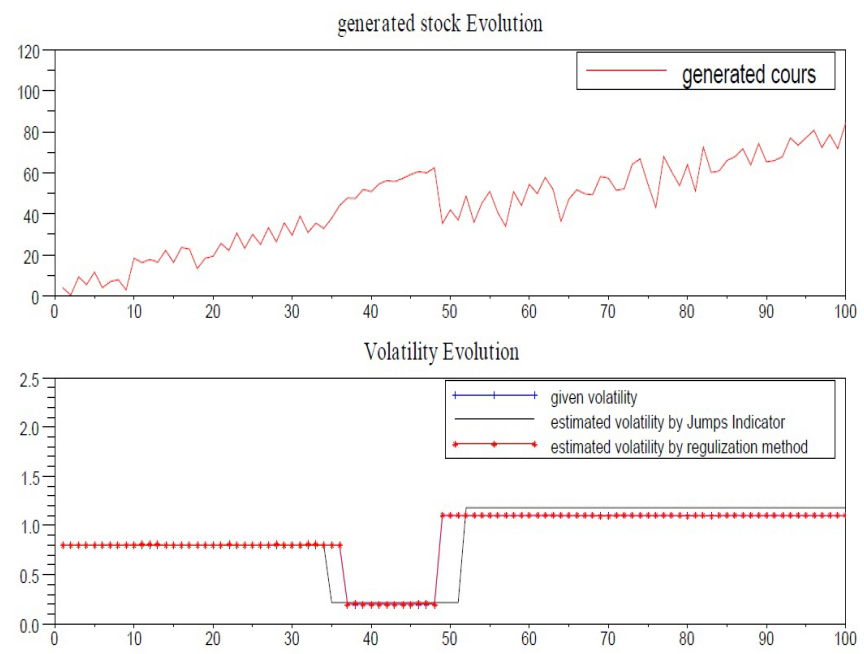

Figure 1. Comparison of the volatility evolution estimated by Jump Indicator and by the regularization method

Table 1. Comparison of the estimated volatility $\sigma$ by Jump Indicator and by the regularization method

\begin{tabular}{cccc}
\hline & Given Volatility & Jumps Indicator & Regularization Method \\
\hline$\sigma_{1}$ & 0.8 & 0.8 & 0.80059 \\
$\sigma_{2}$ & 0.2 & 0.216 & 0.2004 \\
$\sigma_{3}$ & 1.1 & 1.18 & 1.1004 \\
\hline
\end{tabular}

Table 2. Comparing the estimated day of jump $\tau$ by Jump Indicator and by the regularization method

\begin{tabular}{cccc}
\hline & Given Volatility & Jumps Indicator & Regularization Method \\
\hline$\tau_{1}$ & 37 & 35 & 37 \\
$\tau_{2}$ & 48 & 51 & 48 \\
\hline
\end{tabular}

The computation of the indicator gives a first jump on the day 35, two days as error before the exact date while estimated volatility error is around $8 \%$. Detection of the second jump is delayed by 3 days compared to the exact date when the error on the estimate of the volatility is $7 \%$ and the CPU time is 29 seconds. The volatility estimated by the regularization strategy method gives two jumps on the same days of the generated jump, the error on the estimate volatility is $0.5 \%$, and the CPU time is 402 seconds. If the regularization strategy method gives more accurate estimation of the jump day and estimation of the value of the volatility, we note that the Jump Indicator method performs in time where the CPU is twelve times less important. 


\section{$6.2 S \& P 500$}

A second application of the algorithm is searching for jumps during the evolution of S\&P500 between 03/01/2011 and 25/05/2011 using Jump Indicator and ARCH method.
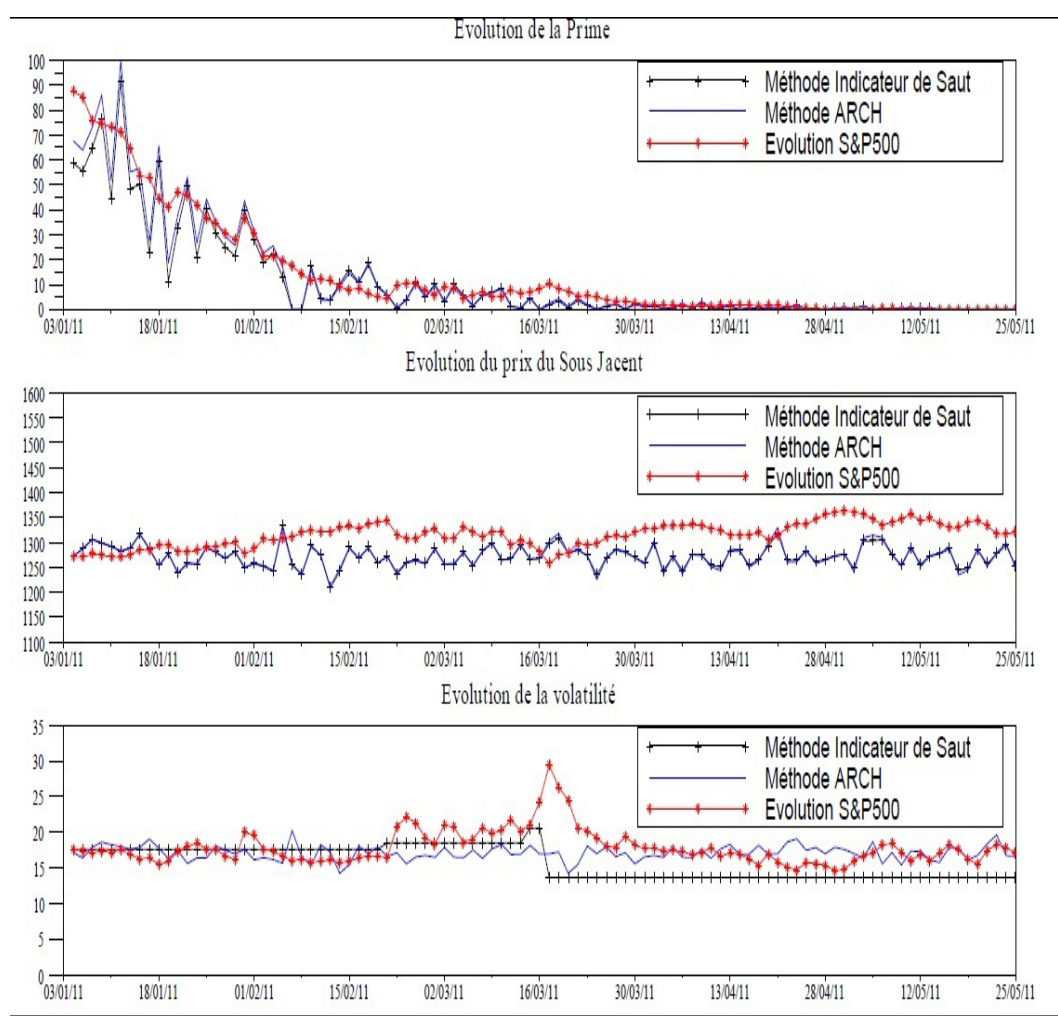

Figure 2. Comparison of the observed volatility in the market and that estimated by Jump Indicator and ARCH

Table 3. Estimated volatility and time of jump using Jump Indicator

\begin{tabular}{cc}
\hline & estimated volatility \\
\hline$\sigma_{1}$ & 17.61 \\
$\sigma_{2}$ & 18.5 \\
$\sigma_{3}$ & 20.57 \\
$\sigma_{4}$ & 13.65 \\
\hline
\end{tabular}

\begin{tabular}{cc}
\hline & estimated jump \\
\hline$\tau_{1}$ & $18 / 02 / 2011$ \\
$\tau_{3}$ & $14 / 03 / 2011$ \\
$\tau_{2}$ & $16 / 03 / 2011$ \\
\hline
\end{tabular}

The first detected jump in volatility occurs on 18/02/2011 which corresponds to four days before the change in the volatility observed in the market, while the second and the third one are estimated on 14/03/2011 and 16/03/2011 which corresponds exactly to a change volatility observed in the Market. The average error for the underlying price and the estimated value for the stock price by the Jump Indicator 42,89 so $3.26 \%$ of the underlying's mean while we obtain 42,72 so $3.25 \%$ of the underlying's mean as average error between the underlying price and the estimated value for the stock price by $\mathrm{ARCH}$ model so the difference between the two errors is around $0.3 \%$ relative of the last one.

\section{Conclusion and Perspective}

In this work, an algorithm for volatility estimation is elaborated using a Jump Indicator. The proposed algorithm is easy to implement, it is also very fast and efficient. Furthermore, it gives the possibility to compute the discontinuities of the volatility in an iterative way.

In (Aboulaich et al., 2012) the prime of an European Lookback Option is calculated using a deterministic model 
and supposing that the volatility is constant. In this paper, we improve this computation estimating a volatility via a jump indicator algorithm and using a more general assumption and supposing that a volatility is piecewise constant function of time. In other hand, we compare the proposed algorithm using a jump indicator with a statistical method ARCH who gives an estimation of historic volatility and with a numerical method based on regularization approach used by (Aboulaich \& Medarhri, 2013) who search for implicit volatility. The numerical results obtained by Jump Indicator algorithm are better compared to those obtained by the ARCH model. The regularization strategy using Dupire's model in (Aboulaich \& Medarhri, 2013) gives more accurate results but it is more expensive.

\section{Acknowledgment}

We would like to thank the Euro Mediterranean (3+3) Hydrinv project and LIRIMA laboratory for their financial support.

\section{References}

Aboulaich,R., Alami Idrissi, A., \& Lamarti Sefian, M. (2012). Simulation of European Lookback Options. IJAMAS, 28(4), 101-117.

Aboulaich, R., \& Medarhri, I. (2013). Calibration of volatility: Coupling between alternative regularization strategyand Dupire's equation. IJAMAS, 42(12), 70-83.

Benameur, H., Chavent, G., Clément, F., \& Weis, P. (2008). The multi-dimensional refinement indicators algorithm for optimal parameterization. Inverse and Ill posed problems, 16, 107-126.

Benameur, H., Chavent, G., \& Jaffré, J. (2002). Refinement and coarsening indicators for adaptive parameterization: application to the estimation of hydraulic transmissitivities. Inverse Problems, 18(3), 775-94.

Bourbonnais, R. (2002). Econométrie. Dunod.

Chiarella, C., Craddock, M., \& el Hassan, N. (2007). The Calibration of Stock Option Pricing Models Using Inverse Problem Methodotogy. School of Finance and Economics, University of Technology Sydney.

Cox, J. C., Ingersoll, J. E., \& Ross, S. A. (1985). An intertemporal general equilibrium model of asset prices. Econometrica, 53, 363-384.

Deberse, M. (2006). Sous-évaluation des prix d'options par le modèle de Black \& Scholes. Mise en évidence par une dynamique combinant mouvement brownien et processus à sauts. Sungard.

Droesbeke, J. J., Fichet, B., \& Tassi, P. (1989). Series Chronologiques. Economica.

Dupire, B. (1994). Pricing with Smile. Risk, 7, 18-20.

Engle, R. (1982).Autoregressive Conditional Htroscedasticity with estimates of the variance of united kingdom inflation. econometrica, 50(4), 987-1008.

Itô, K. (1942). On stochastic processes (infinitely divisible laws of probability). Japan Journal Mathematics, XVIII, 261-301.

Lagnado, R., \& Osher, S. (1997). A technique for calibrating derivative security pricing models: Numerical solution of an inverse problem. The Journal of computational Finance, 1, 13-25.

Merton, R. (1976). Option pricing when the underlying stock returns are discontinuous. Journal of Financial Economics, 3, 125-144.

Tikhonov, A. N. (1963). Regularization of incorrectly posed problems. Soviet Mathematics, 4, 1624-1627.

\section{Copyrights}

Copyright for this article is retained by the author(s), with first publication rights granted to the journal.

This is an open-access article distributed under the terms and conditions of the Creative Commons Attribution license (http://creativecommons.org/licenses/by/3.0/). 